\title{
VIDEOTHORACOSCOPIC SURGICAL TREATMENT FOR NEUROFIBROMA ARISING FROM THE INTRATHORACIC LEFT VAGUS NERVE
} ASSOCIATED WITH RECKLINGHAUSEN'S DISEASE.

\author{
Yasuo Shinoda, Shigebumi Tanaka, Yasumi Yajima, \\ Seiichi Takenoshita, Yukio Nagamachi \\ First Department of Surgery, Gunma University School of Medicine, \\ 3-39-22 Showa-machi, Maebashi, Gunma 371 Japan
}

\begin{abstract}
We report a case of videothoracoscopic surgical treatment of neurofibroma arising from the intrathoracic left vagus nerve associated with Recklinghausen's disease. Tumors that originate in the bilateral vagus nerve are extremely rare. But, as right side, we diagnosed on radiographic identification, not histologically. Only the tumor located near the left vagus nerve was resected due to rapid growth of the tumor. To the best of our knowledge, this is the fourth report of such videothoracoscopic surgical intervention.
\end{abstract}

Key words : Videothoracoscopic surgical treatment, Neurofibroma, Vagus Nerve, Recklinghausen's Disease

(Kitakanto Med.J. 48 (1) : 49 51, 1998)

\section{INTRODUCTION}

Neurofibromas and neurinomas (Schwannomas) are common mediastinal tumors of neurogenic origin that arise from the sympathetic or intercostal nerves in the posterior mediastinum. However, such tumors rarely originate from the vagus nerve ${ }^{1)}$, and the majority of these tumors are encapsulated and benign, solitary and asymptomatic ${ }^{2,3)}$. The present paper reports a method of videothoracoscopic surgical treatment of an intrathoracic left vagal neurofibroma associated with Recklinghausen's disease as well as a review of the available literature on this rare entity.

\section{CASE REPORT}

A 30-year-old Japanese male was transferred to our hospital on August 29, 1996 due to the presence of an abnormal shadow in the mediastinum discovered on his chest $\mathrm{X}$-rays during a routine examination approximately 6 months ago. Multiple subcutaneous nodules were first discovered at the age of 10 , and the patient was diagnosed as Recklinghausen's disease at the age of 27. The patient had no familial history of Recklinghausen's disease. Several nodules on the thoracic wall were painful. The patient was asymptomatic but his condition was complicated with Recklinghausen's disease. Multiple subcutaneous nodules and "cafe-au-

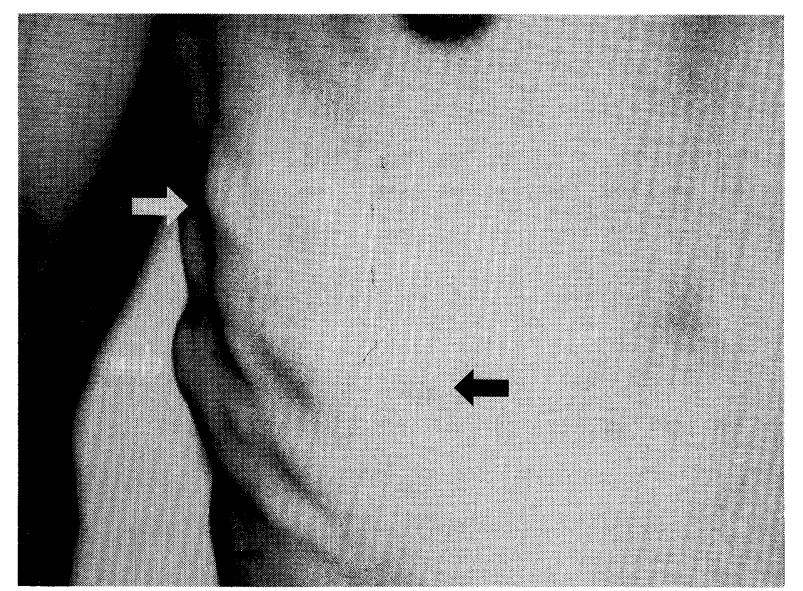

Fig.1. Multiple subcutaneous nodules $(\Rightarrow)$ and "cafe-au-lait" spots $(\leftarrow)$ can be seen over trunk and extremities. 


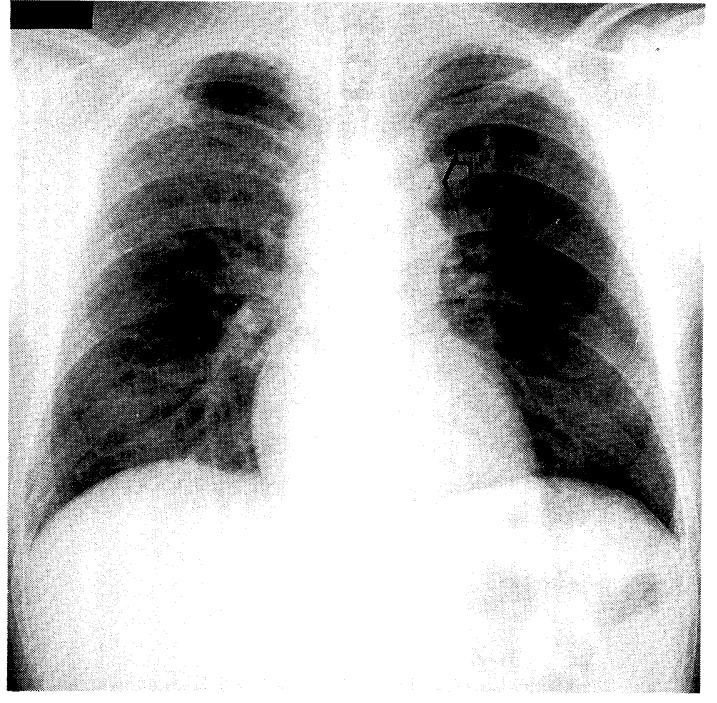

Fig.2. Chest X-rays revealed an upper mediastinal mass( $\diamond)$.

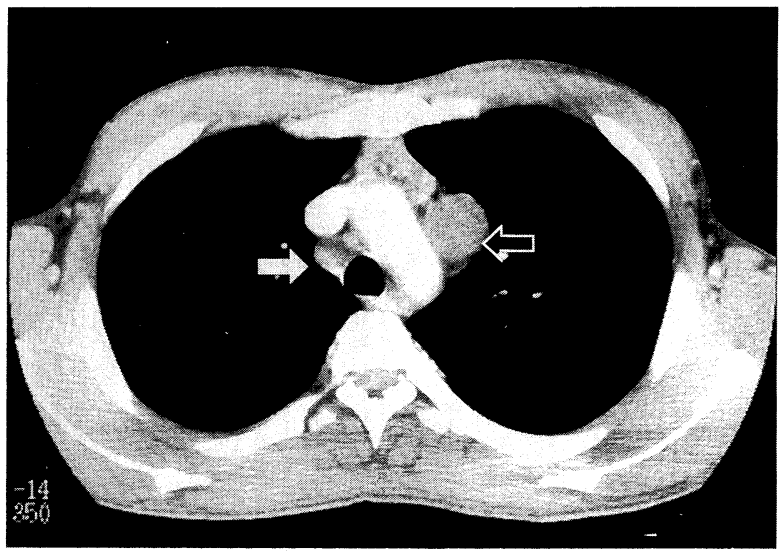

Fig.3. Chest computed tomography (CT) revealed a $4.0 \times 3.0 \mathrm{~cm}$, well-circumscribed, homogenous mass $(\diamond)$ in front of aortic arch in left upper mediastinum and a $1.5 \mathrm{~cm}$ mass on right side $(\rightarrow)$

lait" spots were evident all over his trunk and extremities (Fig.1). No other apparent abnormalities such as hoarseness were evident.

Chest X-rays revealed an upper mediastinal mass (Fig.2). Chest computed tomography (CT) revealed a $4.0 \times 3.0 \mathrm{~cm}$, well-circumscribed, homogenous mass in front of the aortic arch in the left upper mediastinum, as well as a $1.5 \mathrm{~cm}$ mass on the right side (Fig.3). The patient was ultimately diagnosed as a mediastinal tumor arising from the bilateral vagus nerve. Only the tumor on the left side of the vagus nerve was resected due to rapid growth compared with 6 months ago. The tumor on the right side was not resected due to relatively small size as well as the risk of bilateral recurrent nerve paralysis.

We performed videothoracoscopic surgery. Under general anesthesia with unilateral ventilation, the patient was turned in the right decubitus position and we tilt the operative table to allow gravity assistance for the displacement of the lung. A rigid videothor- acoscope was insert through a thoracoport. The tumor was resected using the dissecting scissors with electric cautery through two other thoracoports. The tumor, measuring approximately $4.0 \mathrm{~cm}$ in diameter, was found adjacent to the aortic arch arising from the left main vagus nerve in the antero-superior mediastinum. The upper edge of the tumor was located at the level of the top of the left thoracic cavity, and the lower edge was at the level of the main pulmonary artery. The tumor could be resected easily by transecting the left main vagus nerve (Fig.4). Three subcutaneous nodules in the thoracic region were also resected due to reported pain. The resected tumor was an encapsulated mass that measured $10.0 \times 4.0 \times 3.0 \mathrm{~cm}$. The outer surface was yellowish-gray and the cut surface was homogenous and white-tan in color.

Histologically, the tumor was composed of fibroblasts and Schwann cells with no malignant indication (Fig.5). The mass was determined to be a neurofibroma that originated in the vagus nerve. The thoracic subcutaneous nodules were determined histologically to be the same. At present, approximately six months

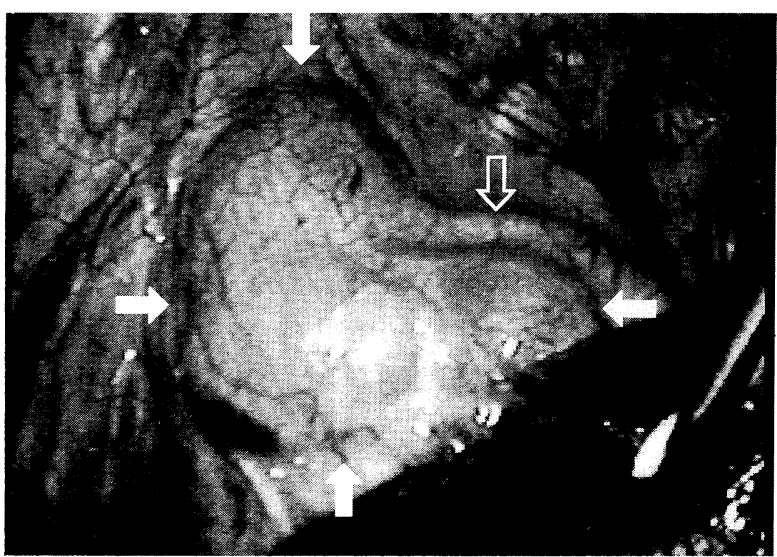

Fig.4. Tumor arising from left main vagal nerve in anterosuperior mediastinum, measuring approximately $4.0 \mathrm{~cm}$ in diameter $(\leftarrow)$, was found adjacent to aortic arch $(\hookleftarrow)$.

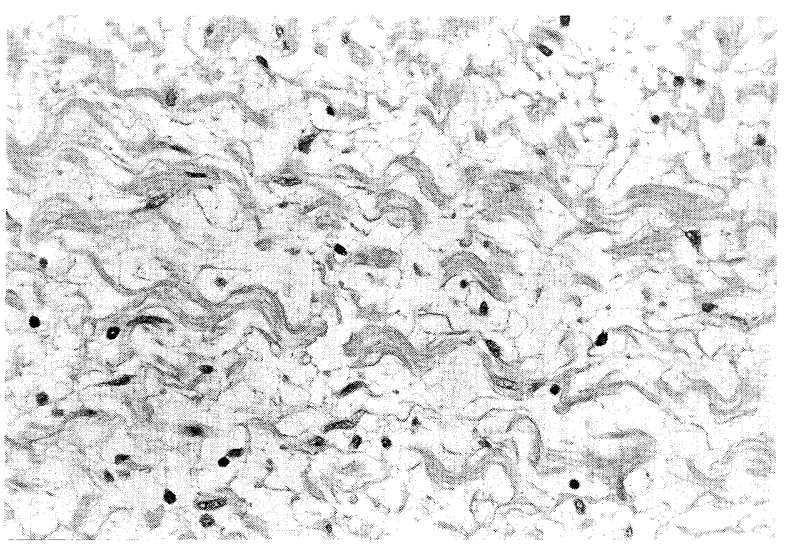

Fig.5. Histologically, tumor was composed of fibroblasts and Schwann cells with no malignant indication (H-E stain, $\times 400$ ). 
has passed since the surgical procedure was performed The postoperative clinical course have been uneventful with the exception of hoarseness due to recurrent nerve paralysis.

\section{DISCUSSION}

Neurogenic tumors are common mediastinal tumor, and generally originate from the sympathetic or intercostal nerves in the posterior mediastinum, whereas few researchers have reported tumors arising from the intrathoracic vagus nerve. Besznyak et al. ${ }^{2)}$ reported only 2 cases of intrathoracic vagal tumors of a total 47 cases of intrathoracic neurogenic tumors reviewed. Heitmiller et al. ${ }^{4)}$ reported that only 3 of his reported 214 intrathoracic neurogenic tumors were of vagal origin.

The Japanese literature includes a total of 49 reported cases of intrathoracic vagal tumors, including the present case. Histologically, 44 cases (89.8\%) were diagnosed as neurinoma (Schwannoma) and 5 cases $(10.2 \%)$ were diagnosed as neurofibroma. Of the 5 neurofibroma cases, 4 cases $(80.0 \%)$ were complicated with associated Recklinghausen's disease. Dabir et al. ${ }^{3)}$ reported that among 27 intrathoracic neurogenic tumors arising from the vagus nerve, 15 (55.6\%) were diagnosed as neurofibromas. Accompanying Recklinghausen's disease was observed in $53.3 \%$ of these neurofibromas. The results of histological studies differ between Japan and the West. Neurinomas tend to be more prevalent in Japan, whereas neurofibromas were more frequently reported in the West. Intrathoracic vagal tumors are almost always benign, and malignant tumors are extremely rare. In Japan, only 2 cases of malignant Schwannoma were reported in 1993. ${ }^{5,6)}$ In the West, only 4 vagal tumors have been reported to be malignant, histologically-confirmed neurofibrosarcoma. ${ }^{2,3)}$ Thus, racial difference in the types of vagal tumors were seen.

Dabir et al. ${ }^{3)}$ reported that of 29 vagal tumors, 18 were located on the left side, 6 were located on the right side, 2 were bilateral, and in 3 instances the author did not specify. In Japanese literature, almost the same results were seen. Thus, location does not tend to differ between the West and Japan. The present case is the first reported incidence in Japan of a neurofibroma arising from the bilateral vagus nerve. As reported, only the tumor of the left vagus nerve was resected. The tumor on the right side will also require treatment in the future.
Videothoracoscopic surgery is a relatively new procedure for treating mediastinal tumors of the thorax. To the best of our knowledge, 3 such procedures have been performed previously for the intrathoracic vagal nerve ${ }^{7 \sim 9)}$. Videothoracoscopic resection of mediastinal tumors is minimally invasive and appears to be more effective than conventional thoracotomy.

Benign vagal tumors are usually asymptomatic and tend to be found incidentally on chest X-rays during routine examination ${ }^{3)}$. In contrast, malignant vagal tumors often advance locally in the mediastinum and lead to various symptoms such as hoarseness, dyspnea and tachycardia ${ }^{3}$. Based on previous reports as well as the results from the present case, surgical resection should be indicated for patients who complain of the above symptoms, or show rapid growth of the tumor.

\section{REFERENCES}

1) Das Gupta TK, Brasfield RD, Strong EW, Hajdu SI. Benign solitary schwannomas (neurilemomas). Cancer 1969 ; 24 : 355-356.

2) Besznyak I, Toth L, Szende B. Intrathoracic vagus nerve tumors: A report of two cases and review of the literature. J Thoracic Cardiovasc Surg 1985 ; 89 : 462-465.

3) Dabir RR, Piccione W Jr, Kittle CF. Intrathoracic tumors of the vagus nerve. Ann Thorac Surg 1990 ; 50 : 494-497.

4) Heitmiller RF, Labs JD, Lipsett PA. Vagal schwannoma. Ann Thorac Surg 1990; 50: 811813.

5) Maebaya S, Miyoshi S, Nishino E. Malignant schwannoma of the intrathoracic vagus nerve: Report of a case. Surgery Today, Jpn J Surg 1993 ; 23 : 1078-1080.

6) Yano T, Hara N, Ohta M. An intrathoracic vagus nerve schwannoma invading the trachea. Surgery Today, Jpn J Surg 1993 ; 23 : 1113-1115.

7) Watanabe M, Takagi K, Tanaka S. Thoracoscopic resection of mediastinal tumors. J Jpn Assn Thorac Surg 1994; 42 : 1016-1020.

8) Riquet M, Mouroux J, Jancovici R. Videothoracoscopic Excision of Thoracic Neurogenic Tumors. Ann Thorac Surg 1995; 60 : 943-946.

9) Nakamura $H$, Araki $K$, Mori $T$ et. A case of thoracoscopic resection for benign schwannoma originating from the vagal nerve. J Jpn Assn Chest Surg 1997; 11 : 90-94. 